\title{
A prática dialógica com pacientes oncológicos: experiência de acadêmicos de enfermagem
}

\author{
Dialogical practice with cancer patients: experience of nursing students \\ Práctica dialógica con pacientes oncológicos: experiencia de estudiantes de enfermería \\ lago Orleans Pinheiro Monteiro ${ }^{1 *}$, Patrícia da Costa Franco ${ }^{1}$, Henry Walber Dantas Vieira ${ }^{1}$, José \\ Jailson de Almeida Júnior².
}

\section{RESUMO}

Objetivo: relatar a experiência vivenciada por acadêmicos de Enfermagem no diálogo traçado durante a coleta de dados de uma pesquisa de doutorado com pacientes oncológicos de diversos setores de um Hospital-referência em tratamento de câncer no estado do Amazonas. Métodos: Trata-se de um estudo descritivo, tipo relato de experiência. Para embasamento metodológico, utilizou-se as etapas de Sistematização de Experiências, proposto por Holliday JO. Relato de Experiência: A experiência foi fruto do diálogo estabelecido no momento da aplicação de instrumentos de coleta de dados aos usuários dos setores de tratamento da Fundação Centro de Controle de Oncologia do Estado do Amazonas localizada no Município de Manaus, Estado do Amazonas, por acadêmicos de Enfermagem da Escola de Enfermagem de Manaus Universidade Federal do Amazonas (EEM/UFAM) ao participarem como colaboradores de uma pesquisa de doutorado. Conclusão: Dentro do processo dialógico a enfermagem torna-se grande operacionalizadora das estratégias de comunicação para a melhor recuperação do indivíduo hospitalizado. Esta realidade deve ser trabalhada no contexto acadêmico, tendo em vista que o diálogo empregado com pacientes, através de práticas executadas ainda na academia resultam na formação de um profissional preparado para lidar com as singularidades dos indivíduos em tratamento de câncer.

Palavras-chaves: Enfermagem, Oncologia, Relações Interpessoais.

\begin{abstract}
Objective: To report the experience of Nursing students in the dialogue drowing during the data collection from a doctoral research with cancer patients from various sectors of a reference hospital in cancer treatment in the state of Amazonas. Methods: This is a descriptive study, type experience report. For methodological basis, we used the stages of Systematization of Experiments, proposed by Holliday JO. Experience Report: The experience was the result of the dialogue established at the time of application of data collection instruments to users of the treatment sectors of the Amazonas State Oncology Control Center Foundation located in Manaus, Amazonas State, by academics. Nursing School of Manaus - Federal University of Amazonas (EEM / UFAM) by participating as collaborators in a doctoral research. Conclusion: Within the dialogical process, nursing becomes a major operationalizer of communication strategies for better recovery of hospitalized individuals. This reality should be worked out in the academic context, given that the dialogue employed with patients, through practices performed still in the academy, results in the formation of a professional prepared to deal with the singularities of individuals undergoing cancer treatment.
\end{abstract}

Key words: Nursing, Oncology, Interpersonal Relations.

\footnotetext{
${ }^{1}$ Universidade Federal do Amazonas/ Escola de Enfermagem de Manaus (UFAM/EEM), Manaus- AM.

*E-mail: orleansiago@hotmail.com

2 Universidade Federal do Rio Grande do Norte (UFRN), Santa Cruz - RN.
} 


\section{RESUMEN}

Objetivo: informar sobre la experiencia de los estudiantes de enfermería en el diálogo dibujado durante la recopilación de datos de una investigación doctoral con pacientes con cáncer de diversos sectores de un hospital de referencia para el tratamiento del cáncer en el estado de Amazonas. Métodos: Este es un estudio tipo descriptivo, informe de experiencia de tipo. Para bases metodológicas, utilizamos las etapas de la Sistematización de Experimentos, propuestas por Holliday JO. Informe de experiencia: La experiencia fue el resultado del diálogo establecido al aplicar instrumentos de recopilación de datos a los usuarios de los sectores de tratamiento de la Fundación Centro de Control de Oncología del Estado de Amazonas, ubicada en Manaus, Estado de Amazonas, por parte de académicos. Escuela de enfermería de Manaus - Universidad Federal de Amazonas (EEM / UFAM) participando como colaboradores en una investigación doctoral. Conclusión: dentro del proceso dialógico, la enfermería se convierte en un operador importante de las estrategias de comunicación para una mejor recuperación de las personas hospitalizadas. Esta realidad debe ser desarrollada en el contexto académico, dado que el diálogo empleado con los pacientes, a través de las prácticas realizadas aún en la academia, da como resultado la formación de un profesional preparado para lidiar con las singularidades de las personas que reciben tratamiento para el cáncer.

Palabras clave: enfermería; Oncología; Relaciones interpersonales

\section{INTRODUÇÃO}

O câncer é denominado a um conjunto de mais de 100 doenças que se configuram pelo crescimento desordenado de células malignas em tecidos e órgãos tendo a possibilidade de fazer metástase (espalharse) para outras regiões do corpo. Para o biênio 2018-2019 estima-se a ocorrência de 600 mil casos novos no Brasil para cada ano e considera-se atualmente a morte de mais de 8,2 milhões de pessoas por ano no mundo (BRASIL, 2019; BRASIL, 2017).

Na região norte, o Instituto Nacional do Câncer (INCA) estimou para o mesmo ano, 34.310 novos casos de câncer, sendo destes, 5.860 no estado do Amazonas e 4.250 na cidade de Manaus. Dentro dessa realidade, a Fundação Centro de Controle de Oncologia do Estado do Amazonas (FCECON), destaca-se atualmente como referência no diagnóstico e tratamento do câncer em toda a Amazônia Ocidental, além de ter destaque com as ações desenvolvidas nas áreas de Prevenção, Ensino e Pesquisa (BRASIL, 2017; FCECON, 2019).

No quadriênio 2010-2014 a FCECON desenvolveu, respectivamente, 4.422 .246 e 101.494 atividades ambulatoriais e hospitalares, além destes, é cabível destacar que os serviços prestados pela enfermagem alcançaram o número de 503.223 de atividades oferecidos a população (FCECON, 2019).

A assistência prestada pela equipe de enfermagem aos pacientes em tratamento de câncer é peça fundamental na amenização do sofrimento vivido pelo indivíduo que enfrenta fragilização emocional e física acarretada pelo desenvolvimento da patologia. Nesse cenário, o enfermeiro deve gerenciar o cuidado embasado em conhecimento técnico-científico adquirido durante sua formação e profissão sobre a doença e como o indivíduo responde a ela, de forma a estabelecer um relacionamento interpessoal que permita a oferta de um cuidado holístico (VICENZI A, et al., 2013).

Na prestação de assistência, é necessário o emprego da interação mútua entre os atores envolvidos (paciente-profissional) no processo de cuidar, assim, a comunicação adotada fornecerá subsídios para a adoção de condutas assertivas relacionadas ao quadro clínico observado. A comunicação é considerada um dos instrumentos base da enfermagem, sendo essencial para o exercício da profissão e, portando deve ser o alicerce para a formação do enfermeiro (VEIGA KCG, et al., 2010; HORTA WA, 1970).

Ademais, é importante salientar que uma possibilidade de aproximação entre pessoas é pelo diálogo, pois, através dele há um contato mais próximo, uma relação de integração de culturas, e uma troca de vivências e experiências (BARRA DCC, et al., 2010). 
O diálogo estabelecido durante as práticas de cuidado no ambiente hospitalar denomina-se como prática dialógica. Esta permite resultados positivos no quadro do indivíduo, pois permite a aproximação entre 0 paciente e a equipe, a troca de experiências, o fortalecimento pessoal do paciente, além de propiciar que o enfermeiro mostre solidariedade com a dor do cliente, efetivando a prática do cuidado integral e holístico da enfermagem (BARCELOS LMS e ALVIM NAT, 2006).

A prática dialógica adotada por enfermeiros frente aos pacientes com câncer é primordial no processo de tratamento, porquanto estabelece elo de confiança que possibilita o profissional identificar as concepções e necessidades do indivíduo, além de se reconhecer como gerenciar do cuidado. Necessidade como resposta aos questionamentos dos pacientes, apoio e companheirismo através de toque, sorriso e expressão de afeto por parte dos profissionais de saúde são demandas que estão na base das relações humanas e podem ser atendidas por meio da prática dialógica (FONTES CAS e ALVIM NAT, 2008).

Considerando as afirmações expostas, para que essas práticas sejam adotadas pelo profissional é viável que haja uma inferência desses conceitos ainda na academia, reafirmando a relevância de tais atitudes na profissão. Dessa forma, este trabalho objetiva relatar a experiência vivenciada por acadêmicos de enfermagem no diálogo traçado durante a coleta de dados de uma pesquisa de doutorado com pacientes oncológicos de diversos setores de um Hospital-referência em tratamento de câncer no estado do Amazonas.

\section{MÉTODOS}

Trata-se de um estudo descritivo, tipo relato de experiência. Para embasamento metodológico, utilizou-se as cinco etapas de Sistematização de Experiências, proposto por Holliday JO (2006). A primeira etapa é denominada "Ponto de partida" para sua concretização é necessário que o autor tenha vivenciado a experiência e tenha os registros organizados.

O segundo momento, as "Perguntas iniciais", são as responsáveis pela delimitação da experiência, objeto da sistematização, assim como, o objetivo e o eixo que será considerado dentre os vários aspectos da experiência. Para este artigo, considerou-se como objeto, a experiência durante uma coleta de dados de uma pesquisa e como eixo, a prática dialógica.

$\mathrm{Na}$ terceira etapa, denominada "Recuperação do processo vivido", descreve-se todo o processo vivenciado. Na quarta etapa, "Reflexão de fundo", procura-se responder a questão "Por que aconteceu o que aconteceu?", para isso, considera-se a busca de respostas na literatura, momento em que se descrevem e discute-se os resultados alcançados.

Ainda nesta etapa, considerou-se relevante a divisão da discussão em duas categorias - "O fazer pesquisa como mecanismo indutor de vivências em novos cenários da saúde" e "O processo dialógico como benefício à saúde do paciente e facilitador do processo de cuidado".

Na última etapa "Ponto de chegada", é o momento onde chega-se às conclusões acerca dos aspectos da experiência que podem influenciar na atuação de outros em experiências similares. Torna-se necessário que na última etapa haja a divulgação dos resultados, fato concretizado com a publicação deste artigo.

\section{Recuperação do processo vivido}

A experiência foi fruto do diálogo estabelecido no momento da aplicação de instrumentos de coleta de dados aos usuários dos setores de tratamento da Fundação Centro de Controle de Oncologia do Estado do Amazonas localizada no Município de Manaus, Estado do Amazonas, por acadêmicos de Enfermagem da Escola de Enfermagem de Manaus - Universidade Federal do Amazonas (EEM/UFAM) ao participarem como colaboradores de uma pesquisa de doutorado.

A vivência ocorreu no período de abril a julho de 2016 através da etapa de coleta de dados referente à pesquisa intitulada "Validade de constructo do Supportive Care Needs Survey - Short Form 34 (SCNS-SF34) para a avaliação de cuidados de pacientes oncológicos atendidos em Manaus" de um docente da EEM/UFAM, doutorando do curso de Pós-Graduação em Enfermagem na Saúde do Adulto em parceria com a Escola de Enfermagem de São Paulo (EEUSP). 
Foram selecionados acadêmicos voluntários que cursavam períodos diferentes do curso de Enfermagem para colaborarem no estudo com intuito de suprir, em tempo hábil, a demanda mínima exigida para a amostra da pesquisa. Salienta-se que foi realizado treinamento introdutório que objetivou apresentar a proposta da pesquisa, capacitá-los para a abordagem ao paciente e aplicação do instrumento de coletas de dados.

A atividade consistiu na aplicação de um instrumento com os pacientes dos setores de quimioterapia e enfermaria. Cada acadêmico ficou responsável igualitariamente por um número específico de dados a serem coletados, sendo o pesquisador o responsável pelo maior quantitativo dos mesmos.

Para execução da coleta, os acadêmicos apresentaram aos pacientes a proposta da pesquisa, bem como, o Termo de Consentimento Livre e Esclarecido que deveria ser assinado, dessa forma, respaldando o pesquisador quanto ao consentimento do usuário em participar da pesquisa e assegurando-o do sigilo das informações prestadas.

O prosseguimento da atividade permitiu estabelecer um diálogo que possibilitou a obtenção das informações requeridas no instrumento. Para que todos os dados fossem fielmente obtidos, o processo dialógico se estabelecia no tempo do paciente, durando cerca de quarenta e cinco minutos.

Para além, as informações complementares, no que tange ao processo patológico e tratamento, eram obtidas através da permissão do paciente à consulta ao prontuário disponível no posto de enfermagem dos setores.

\section{RESULTADOS E DISCUSSÃO}

\section{Reflexão de fundo}

\section{O fazer pesquisa como mecanismo indutor de vivências acadêmicas dialógicas em enfermagem}

A Enfermagem ultrapassou um grande processo histórico até chegar ao que se tem hoje como profissão e ciência. Os primeiros movimentos científicos na enfermagem passam a ocorrer com a percussora da profissão na modernidade, Florence Nightingale, figura importante que utilizou de artifícios da época em que viveu para consolidar a prática dos cuidados de forma mais padronizada, considerando o cuidado para além dos aspectos individuais dos doentes (BARBOSA D, et al., 2014).

Entretanto, somente no ano de 1900 surge a primeira publicação no American Journal of Nursing, passando a ser a prática de pesquisa essencial para a atuação na prestação de cuidados de saúde, dessa forma, busca-se atualmente na produção científica a melhor prática a ser considerada para o trabalho em enfermagem (BARBOSA D, et al., 2014).

Neste contexto, observa-se a grande relevância que a pesquisa tem para a consolidação da profissão, sendo imprescindível que a aplicabilidade do contexto cientifico ocorra desde a formação até a atuação profissional, de forma contínua, pois, a trajetória acadêmica somada a prática científica resulta em qualidade na formação do enfermeiro (CANTíDIO FS, et al., 2011; DOMENICO EBLD e IDE CAC, 2003).

Ademais, apesar do impacto que a produção de evidências científicas pode trazer para a profissão nos diversos contextos, qual o papel que a prática da pesquisa com indivíduos exerce no meio em que se aplica?

Dentre os benefícios que poderiam ser listados, considera-se a partir da experiência vivida, que o diálogo exercido durante o seguimento da coleta de dados tornou-se uma ferramenta de comunicação eficaz, permitindo tanto aos acadêmicos entrevistadores executarem a prática dialógica, quanto aos entrevistados a possibilidade de serem ouvidos com mais atenção.

Essa dinâmica traçada a partir da entrevista resulta em um retorno diversificado para o entrevistado, tendo em vista a gratificação emocional que o método traz, pelo fato de ter participado de uma interação em que este foi o centro das atenções, além da gratificação cognitiva, que se deve ao fato de ter concluído um bom trabalho sobre si próprio. Além disso, a entrevista em pesquisa, possibilita ao entrevistado a exposição de suas singularidades à alguém que não conhece, dessa forma, o prepara para que tenha um melhor contato com a equipe que vai lhe prestar cuidados em saúde, que por vezes, ele nunca viu (CARDANIO M, 2017). 


\section{O processo dialógico como benefício à saúde do paciente oncológico}

Considerando que o relacionamento Enfermeiro/paciente pode trazer grandes benefícios para o processo de cuidado e consequente evolução positiva do quadro do cliente, a utilização desta técnica se torna terapêutica. Somado a isso, o sucesso deste cuidado, também se dá pela experiência prévia dos profissionais.

Portanto, experienciar o relacionamento com o paciente oncológico em uma perspectiva dialógica, potencializou a capacidade dos acadêmicos para atuarem frente às problemáticas encontradas no cuidado de enfermagem em oncologia (VEIGA KCG, et al., 2010).

Dessa forma, buscam-se técnicas para proceder nesse relacionamento terapêutico, sendo o diálogo constatado, a partir da experiência, como uma ferramenta eficaz, tendo em vista, a exposição dialógica dos sujeitos sobre os variados impactos que a doença trouxe para suas vidas, fruto do contato no momento da pesquisa.

Assim, a participação em etapas da pesquisa, tornou-se uma estratégia que possibilitou o contato dos discentes nessa realidade, fornecendo meios de planejamento para uma prática futura, reafirmando a relevância de ouvir o paciente para o delineamento de um plano de cuidados eficaz.

A prática dialógica foi a máxima vivenciada nesta experiência e a grande satisfação dos estudantes foi por poder vivencia-la em um campo, até então, pouco explorado durante a graduação, o cuidado em oncologia. As realidades, experiências, dificuldades, crenças e atitudes abordadas pelos clientes de forma espontânea e aberta mostraram a necessidade que estes tinham em poder dialogar e expressar seus sentimentos.

Dialogar com os indivíduos é uma prática natural exercida pelo ser humano, a partir deste contato ocorrem as trocas e relações e justamente essa é a necessidade que doentes hospitalizados por longos dias e/ou em tratamento no meio hospitalar encontram.

Executar esta prática permite um novo olhar e melhor aproveitamento da relação paciente-profissional, além de inúmeros benefícios que culminam na melhora do quadro patológico instalado (BARCELOS LMS e ALVIM NAT, 2006; SALIMENA AMO, 2013).

Por vezes, o diálogo é abordado durante o percurso da graduação em enfermagem como sendo uma ferramenta eficaz para o cuidado, todavia, na prática no ambiente hospitalar, a rotina de atendimento não permite tal exercício.

Atividades de extensão que permitem o contato com a comunidade e indivíduos de forma mais próxima, podem ser meios para operacionalizar essa prática ainda no meio acadêmico, com vista a formação de profissionais mais comprometidos com a realidade das diversidades sociais, logo, profissionais que compreendem os indivíduos em suas subjetividades (FERREIRA MR, et al., 2012).

\section{CONCLUSÃO}

O processo dialógico configura-se como um impacto positivo para o cuidado à pacientes oncológicos. A equipe de enfermagem se torna grande operacionalizadora das estratégias de comunicação para a melhor recuperação do indivíduo hospitalizado. Essa realidade deve ser trabalhada no contexto acadêmico, tendo em vista que o diálogo empregado com pacientes, através de práticas executadas ainda na academia resultam na formação de um profissional preparado para lidar com as singularidades dos indivíduos, disposto a ouvir suas necessidades, dificuldades, experiências, crenças e anseios. Compreender esses fatores potencializará a gestão de cuidados holísticos e integrais pelo futuro enfermeiro.

Portanto, a experiência vivenciada por acadêmicos de enfermagem na coleta de dados de uma pesquisa científica em contexto oncológico hospitalar reafirmou o impacto que a pesquisa pode ter para além dos resultados e evidências científicas que podem gerar. Espera-se que este artigo cause uma reflexão acerca dos aspectos referentes a pesquisa científica como mediadora da prática dialógica para elaboração de estudos que comprovem esta realidade em aspectos metodológicos e que a experiência seja replicada em outros meios acadêmicos. 


\section{REFERÊNCIAS}

1. BARBOSA D, TAMINATO M, FRAM D, BELASCO A. Enfermagem Baseada em Evidências. 1 ed. São Paulo: Editora Atheneu, 2014.

2. BARCELOS LMS, ALVIM, NAT. Atenção e presença física: dimensões expressivas e a prática dialógica do cuidado de enfermagem na perspectiva do cliente hospitalizado. Rev Bras de Enferm, 2006; 59(1): 25-9.

3. BARRA DCC, et al. Hospitalidade como expressão do cuidado em enfermagem. Rev Bras Enferm, 2010; 63(2):2038.

4. BRASIL. Instituto Nacional de Câncer José Alencar Gomes da Silva. Coordenação de Prevenção e Vigilância. Estimativa 2018: incidência de câncer no Brasil. Rio de Janeiro: INCA, 2017. 128 p.

5. BRASIL. Instituto Nacional de Câncer José Alencar Gomes da Silva; organização Mario Jorge Sobreira da Silva. ABC do câncer: abordagens básicas para o controle do câncer. Rev Atual. Ampl. Rio de Janeiro: INCA, 2019, 5 ed. $111 \mathrm{p}$.

6. CARDANIO M. Manual de Pesquisa Qualitativa: A contribuição da teoria da argumentação. Rio de Janeiro: vozes, 2017.

7. CANTíDIO FS, et al. Significado da morte e de morrer para os alunos de enfermagem. Invest. Educ. enferm. 2011; 29(3):407-18.

8. DOMENICO EBLD, IDE CAC. Enfermagem baseada em evidências: princípios e aplicabilidades. Rev Latino-am Enfermagem, 2003; 11(1):115-8.

9. FCECON-Fundação Centro de Controle de Oncologia do Estado do Amazonas. Governo do Estado do Amazonas. Disponível em: https:// www.fcecon.am.gov.br. Acesso em: 20 jul. 2019.

10. FERREIRA MR, et al. Da dialogicidade entre universidade e comunidade: um estudo de caso da extensão universitária a partir do exercício da democracia dialógica na pesquisa-ação. Cadernos Gestão Social, 2012; 3(1):5368.

11. FONTES CAS, ALVIM NAT. Cuidado humano de enfermagem a cliente com câncer sustentado na prática dialógica da enfermeira. Rev. enferm. UERJ, 2008; 16(2):193-199.

12. HOLLIDAY OJ. Para sistematizar experiências. 2. Ed. Brasilia: MMA, 2006

13. HORTA, WA, et al. O ensino dos instrumentos básicos de enfermagem. Rev. Esc. Enferm. USP, 1970; 4(1):5-20.

14. SALIMENA AMO, et al. O vivido dos enfermeiros no cuidado ao paciente oncológico. Cogitare Enferm. 2013; 18(1):142-7.

15. VEIGA KCG, et al. Relacionamento Enfermeira/Paciente: Perspectiva Terapêutica do Cuidado. Revista de Enfermagem da UERJ. 2010; 18(2): 322-5.

16. VICENZI A, et al. Cuidado integral de Enfermagem ao paciente oncológico e à família. Revista de Enfermagem da UFSM. 2013; 3(3):49-17. 